\title{
A novel microfluidic wound model for testing antimicrobial agents against Staphylococcus pseudintermedius biofilms
}

\author{
Jacob Terry and Suresh Neethirajan*
}

\begin{abstract}
Background: Current methods for testing treatments for veterinary surgical site infections can successfully emulate elements of a chronic wound, but these are time consuming and costly, requiring specialized laboratory equipment and considerable space to house study animals. Microfluidic devices however, can be coated with collagen and maintained at basal body temperature, providing a more cost-effective and space-saving model of a chronic wound. Our study assesses the applicability of a new microfluidic model by testing the activity of DispersinB against biofilms of methicillin-resistant Staphylococcus pseudintermedius (MRSP); DispersinB has been shown to prevent biofilm growth of Staphylococcus epidermidis, another prominent wound colonizer.

Results: We successfully developed a microfluidic model to examine the effects of antimicrobial therapy on biofilms formed by organisms associated with wound infections in companion animals (e.g. MRSP). Although, we were unable to recapitulate previous findings that DispersinB-Gentamycin is highly effective against Staphylococcal biofilms using this model, we were able to confirm its effect in a microtitre plate. Differences in the experimental conditions likely account for this result (e.g. strains tested, flow conditions, treatment time, etc.). In the microtitre plate assay, DispersinB inhibited biofilm growth after a 24 hour period; there was an inverse relationship between the concentration of DispersinB-Gentamycin and the amount of biofilm remaining following treatment. Collagen-coated microtitre plates showed a similar result, but this did not correlate as well; collagen, the most abundant protein in the body may help to retain the biomass of treated biofilms.

Conclusions: Our model may be useful in examining the effect of treatment on wound infections, although we acknowledge that in this model the test organisms may be more recalcitrant to antimicrobials than in other published systems. We contend that this may in fact better represent the conditions in vivo, where organisms associated with chronic wound infections are highly resistant to antimicrobials.
\end{abstract}

Keywords: Microfluidics, Biofilms, Staphylococcus pseudintermedius, Wound model, Antibiofilm

\section{Introduction}

Methicillin-resistant Staphylococcus pseudintermedius (MRSP) bacterial biofilms have rapidly emerged as a serious complication in surgical site and wound infections in companion animals [1]. MRSP infections are being reported with increasing frequency in veterinary hospitals and have become the leading cause of pyoderma and surgical site infections in dogs [1,2]. MRSP infections are of tremendous concern in companion

\footnotetext{
*Correspondence: sneethir@uoguelph.ca
BioNano Laboratory, School of Engineering, University of Guelph, Guelph, ON

* Correspondence: sneethir@uoguelph.ca
BioNano Laboratory, School of Engineering, University of Guelph, Guelph, ON N1G 2 W1, Canada
}

animals because they are challenging to eradicate, as they are typically recalcitrant to traditional antimicrobial therapy [1-3]. Therefore, it is imperative that the foundations of antimicrobial resistance be better understood.

Antimicrobial treatments may be tested in chronic animal wound infections on living animals, but this can lead to undesired consequences if the treatment were to have adverse effects, which may actually worsen infection or the presentation of the wound [1-4]. One way to avoid in vivo studies in veterinary hospitals would be to simulate a wound using synthetic material where possible [5]. Ideally, such models would display all the characteristics of an animal wound without having to use the 
animal as a test subject [2]. Some studies have been conducted that look at this possibility [6-8], but they primarily use materials to simulate the environment without necessarily simulating the wound itself. Finding a method that increases the accuracy of replicating wound tissue, which at the same time is inexpensive and saves both space and materials, would be most desirable. Such a model could emulate a true wound environment, while remaining easily deployable and could be repeated a sufficient number of times to facilitate advanced statistical analysis and model prediction.

Microfluidic technology is a relatively new field that can be applied to studying, evaluating, and understanding primary biofilm growth characteristics, ideally as a means to improve antimicrobial therapies that target pathogenic biofilms [9]. Microfluidic-based wound models can overcome the drawbacks associated with animal studies in testing antimicrobial agents, such as variability and lack of reproducibility. Microfluidics' well-controlled reaction conditions, accurate delivery of drugs, and lower dead volumes will enable the development of novel skin models for testing antimicrobial agents against biofilms in a wound setting. Studies have shown that copious amounts of biofilm will readily form in channels of microscopic size [10], which can allow researchers to study biofilm growth in a confined, controllable, and reproducible space. For example, models with small channel volumes and high surface areas can be used to mimic the spatial environment of a blood vessel [11]. Microfluidic technology can be applied to more precisely understand the evolution of antimicrobial resistance in biofilms by creating dynamic concentration gradients, such as those found at sites where biofilms form [12,13]. Microfluidics can also be used to study the spatial and temporal growth of microorganisms, as well as motility and chemotaxis $[12,13]$. In this study we investigated the efficacy of an antimicrobial agent against MRSP biofilms in a wound model we developed using microfluidic platforms.

DispersinB, a glycoside hydrolase enzyme, has been shown to successfully inhibit biofilm growth in Staphylococcus species, specifically S. epidermidis [14-16]. This suggests that DispersinB may be an effective candidate for testing the viability of new wound infection models; these results can then be verified using established microtitre plate assay methods $[15,17]$. The efficacy of DispersinB has been investigated using conventional techniques for studying wound-associated bacteria, such as Klebsiella penumoniae and Staphylococcus epidermidis [18]. However, its effect on MRSP, another key wound infection-causing bacterium, has not been investigated. At wound sites, a range of multidrug resistant pathogens can be encountered, resulting in tremendous morbidity, mortality, and healthcare costs for both animals and humans. Biofilm formation has been hypothesized as the reason for the emergence of highly resistant and pathogenic MRSP clones [19].

Therefore, we examined the antimicrobial resistance profiles of MRSP biofilms by evaluating the efficacy of DispersinB in combination with Gentamycin in our newly developed model. Collagen coated polymeric microfluidic platforms, coupled with microscopy, enabled the in situ observation of antimicrobial susceptibility and the evolution of distinct microbial morphologies over the course of biofilm formation. Our results helped to determine an effective minimal concentration of DispersinB and Gentamycin that may be sufficient to eradicate MRSP biofilms. Ultimately, this microfluidic assay may be used to improve the clinical management of wound associated biofilm infections in companion animals.

\section{Materials and methods}

\section{Bacterial strains and antimicrobial agent}

The study bacteria chosen for use in this investigation were selected based on data from a previous study of clarithromycin efficacy against MRSP biofilms [1]. The three strains chosen were selected to demonstrate efficacy of DispersinB with gentamicin against bacteria of varying adherence capabilities. High, medium, and low adherence capability were represented by the A12, A92, and SP102 isolates, respectively. The mixture of Disper$\sin B$ and gentamicin used was $1 \mathrm{mg} / \mathrm{mL}$ gentamicin and $200 \mu \mathrm{g} / \mathrm{mL}$ DispersinB, which was suspended in $50 \mathrm{mM}$ sodium phosphate (pH 5.8) and $100 \mathrm{mM} \mathrm{NaCl}$ (Kane Biotech Inc., Winnipeg, Canada).

\section{Culture and dye methods}

Each strain was sub-cultured from frozen stock onto Columbia agar plates with $5 \%$ sheep blood and grown for 24 hours at $35^{\circ} \mathrm{C}$. For microfluidic tests, test tubes containing $5 \mathrm{ml}$ tryptic soy broth with $1 \%$ glucose (TSB-G) were inoculated with A92 isolates to the equivalent turbidity of a 0.5 McFarland standard, then incubated for 3 hours at $35^{\circ} \mathrm{C}$ and diluted with $5 \mathrm{ml}$ of sterile water. Six micro centrifuge tubes were filled with $1 \mathrm{ml}$ of the diluted solution and centrifuged for 3 minutes at $12,000 \mathrm{rpm}$. The supernatant was removed and discarded, and the cells were suspended in an additional $1 \mathrm{ml}$ of water, and then centrifuged again. This washing process was repeated a total of three times. To make the dye solution, $3 \mu \mathrm{L}$ of dimethyl sulfoxide with $1.67 \mathrm{mM}$ SYTO 9 dye and $1.67 \mathrm{mM}$ propidium iodide was suspended in $1 \mathrm{ml}$ water. Two hundred $\mu \mathrm{L}$ of this dye solution was added to the washed cells (currently suspended in $1 \mathrm{ml}$ water) to yield $1.2 \mathrm{ml}$ of dyed cell solution. Tubes were then stored in the dark at room temperature for 30 minutes before use. 


\section{Preparation of microfluidic devices}

Devices used in these experiments were a simple Y-channel device with 3 -in-1 units, with a $3 \mathrm{~mm}$ wide main channel, $1 \mathrm{~mm}$ tall inlet and outlet channels, and a channel height of $400 \mu \mathrm{m}$, providing a total channel volume of $60 \mu \mathrm{l}$. The devices used for the final experiments were coated with $75 \mu \mathrm{g} / \mathrm{ml}$ rat-tail collagen, type I in $0.05 \mathrm{M}$ hydrochloric acid by filling through the outlet channel. After $2 \mathrm{~h}$, the device was aspirated, washed with sterile water, and left to dry at room temperature for $1 \mathrm{~h}$ before use.

\section{Bacterial flow and incubation}

Five hundred $\mu \mathrm{L}$ of the dyed bacterial solution was added to a glass syringe. The device was mounted onto a microscope, and the syringe was connected via tubing with Luer tapers. An outlet tube was connected to collect any extra liquid. Using a syringe pump, bacteria were flowed at a rate of $200 \mu \mathrm{l} / \mathrm{h}$ through the middle channel for $1 \mathrm{~h}$, while the top and bottom inlet reservoirs were sealed using Luer-compatible syringes or seals (Figure 1A). Images were taken of the bacteria flow in the outlet channel every minute during the bacterial flow process. Tubing was disconnected and the device was incubated for $4,8,12$ or 24 hours at $35^{\circ} \mathrm{C}$. Refer to Figure $1 \mathrm{~B}$ for an illustration of the operation of our microfluidic device.

\section{Efficacy testing of dispersin B and/or sterile water}

After the device was incubated, $500 \mu$ lof sterile water was added to a glass syringe and $500 \mu \mathrm{l}$ of either DispersinB or

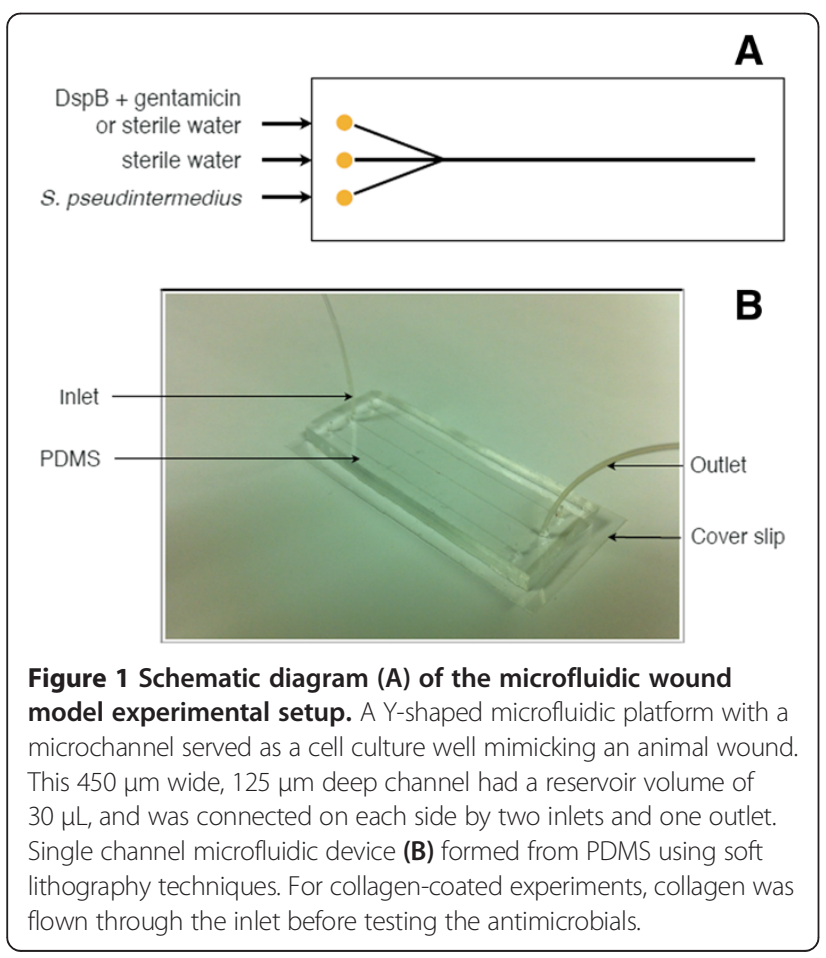

sterile water was added to a second glass syringe. The device was mounted onto a microscope and the syringes were connected via tubing with Luer tapers. An outlet tube was connected with a micro-centrifuge tube to collect the final solution for enumeration testing. Using a syringe pump, DispersinB or sterile water was flowed at a rate of $100 \mu \mathrm{L} / \mathrm{h}$ through the top channel, and sterile water was flowed at a rate of $100 \mu \mathrm{l} / \mathrm{h}$ through the bottom channel for 4 hours, while the middle inlet reservoir was sealed using a Luer-compatible syringe or seal (Figure 1B). Images were taken every minute for the first $2 \mathrm{~h}$ at the same location in the outlet channel that was used.

\section{Enumeration testing}

Micro centrifuge tubes were used to collect liquid suspensions of cells from the outlet tube; serial dilutions of these solutions were made to enumerate the collected cells. We plated the 1:10, 1:100, and 1:1,000 dilutions onto Columbia agar with $5 \%$ sheep blood. The plates were incubated at $35^{\circ} \mathrm{C}$ for $24 \mathrm{~h}$ before the colonies were counted.

\section{Image analysis}

Bacterial flow and post-incubation images were numbered in sequence prior to image analysis. Using MATLAB Version R2013B (Mathworks Inc., Natick, MA), images were read in order and Otsu thresholding was performed to determine fluorescent intensity and surface coverage. To numerically justify the results from the image analysis readings, the area under the curve formed in all surface coverage and intensity graphs was measured and compared.

\section{Micro-titre plate assay}

Epidemiologically unrelated MRSP isolates from dogs from different geographic regions were screened for biofilm production via microtiter plate assay (MPA). Briefly, overnight cultures were suspended in $5 \mathrm{ml}$ of tryptic soy broth (TSB) supplemented with $1 \%$ glucose to achieve a turbidity equivalent to a 0.5 McFarland standard $\left(\sim 10^{8} \mathrm{CFU} / \mathrm{ml}\right) .200 \mu \mathrm{l}$ of each inoculum was transferred in triplicate to a 96-well polystyrene microtiter plate and incubated under aerobic conditions for $24 \mathrm{~h}$ at $35^{\circ} \mathrm{C}$. Following incubation, the plates were washed three times with phosphate buffered saline (PBS) to remove non-adherent cells and then heat fixed at $60^{\circ} \mathrm{C}$ for 60 minutes. Adhered cells were dyed with $0.1 \%(\mathrm{w} / \mathrm{v})$ of crystal violet for 15 minutes and air dried at room temperature. After resolubilization with 95\% ethanol, optical density (OD) reading of each well of the microtiter plate was assessed, taken at $570 \mathrm{~nm}$ (OD570). Readings of replicates for each isolate were averaged and subtracted from the OD570 reading of the negative control. OD570 was used as indication of biofilm production. Isolates were 
classified as biofilm producers if OD570 was $>0.200$ and further classified as strong, moderate, weak, or zero biofilm formers based on their final OD570 reading.

\section{Results and discussion}

We monitored biofilm coverage inside the microfluidic device after 4, 8, 12, and $24 \mathrm{~h}$ of biofilm growth. We began monitoring at each of these time points and performed constant monitoring (images taken every minute) over a $2 \mathrm{~h}$ period. When the percent surface area was calculated and the readings were graphed, similar patterns in the structure of the graphs were observed. We focused on the results obtained from 12 and $24 \mathrm{~h}$ biofilms, as these time points should yield mature structures consistent with chronic wound infections. Surface coverage readings for all four time points showed that $25-50 \%$ of the surface was consistently covered with biofilm (Figure 2A, 2B, 2C and $2 \mathrm{D}$ ) and that relatively stable levels of coverage were maintained over the $2 \mathrm{~h}$ imaging period.

The intensity of biofilm growth in regions across the channel was parabolic as expected, since bacteria flowed through the middle channel before incubation. Unexpectedly, when DispersinB-Gentamycin was used instead of water in the top channel, it was found that the biofilm intensity was greater on average. The average normalized intensity value for $24 \mathrm{~h}$ biofilms was 0.536 for DispersinBGentamycin and 0.500 for water alone, while at $12 \mathrm{~h}$ biofilms it was found to be 0.599 for DispersinB-Gentamycin and 0.411 for water alone. We propose that the apparent increase in biomass may be a response to the stress induced by treatment with antimicrobials. It is not uncommon for the biofilm architecture to be altered in the presence of inimical forces. For example, shear forces applied to biofilm have been shown to result in an increase biofilm density [20].
Comparing the top half of the channel (Figure 3A) with the bottom half of the channel (Figure 3B), we noted that there was a great deal of contrast between these images. All of our experiments produced data with a bias towards the top half of the channel (more biomass is located in this area), indicating that the design of the microfluidic device or the biofilm itself may favour growth on that side of the device. After $24 \mathrm{~h}$, the top half of the channel held an average normalized value of 0.747 when DispersinB-Gentamycin (Figure 3A) or water alone (Figure 3B) was used, while the bottom half held an average normalized value of 0.324 when Disper$\sin \mathrm{B}$ was used and 0.253 when water alone (Figure $3 \mathrm{~B}$ and 3D) was used. After $12 \mathrm{~h}$, the top half of the channel held an average normalized value of 0.765 when DispersinB-Gentamycin was used (Figure 3C) and 0.499 when water was used, while the bottom half held an average normalized value of 0.434 when DispersinBGentamycin was used and 0.322 when water alone was used. These results indicated that there was a greater accumulation of biofilm in the top channel. Again, the accumulation (or lack of dispersion) was enhanced when the biofilms were treated with DispersinB-Gentamycin, which as suggested earlier may be due to a biofilm-promoting response to treatment.

Microtitre plate assay readings confirmed previous reports showing that DispersinB may be effective against wound infection biofilms. We determined that there was an inverse relationship between the DispersinB-Gentamycin concentration and the level of biofilm growth in uncoated wells (Figure 4A). There was also a relationship between the effectiveness of DispersinB-Gentamycin and the biofilm forming capabilities of the selected strain; better biofilm forming strains showed a greater percent reduction in the biomass following treatment. MRSP strain A12 was
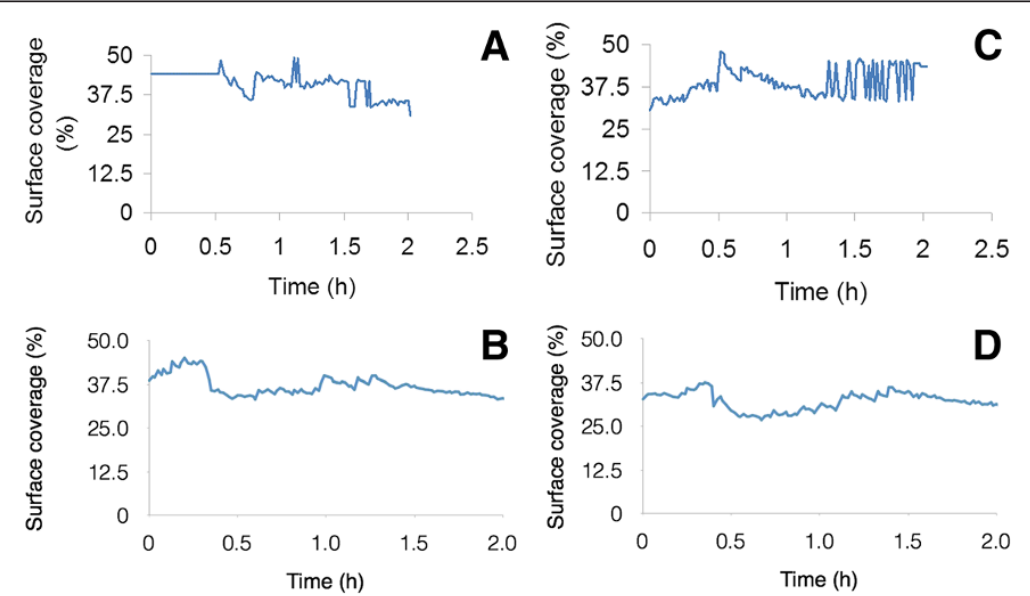

Figure 2 Biofilm coverage inside the microfluidic channels. This figure demonstrates the biofilm surface coverage during a period of 2 hours after each of the denoted time points were reached, using water and DispersinB treatments. Panels are as follows: (A) 24 hours with DispersinB and water, (B) 24 hours with water, (C) 12 hours with DispersinB and water and (D) 12 hours with water. 

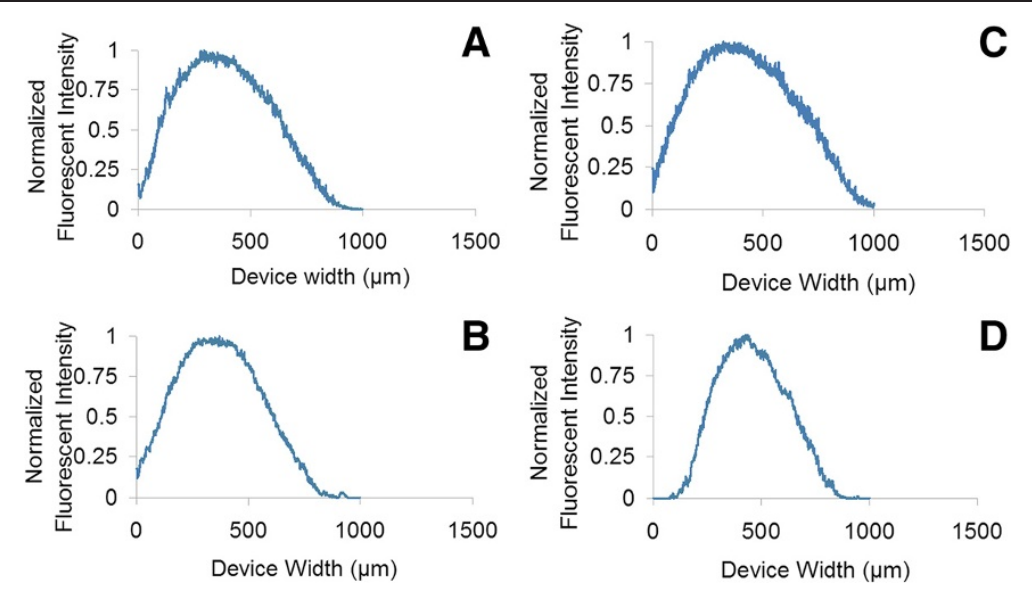

Figure 3 Biofilm fluorescence intensity inside the channels. This figure demonstrates the average fluorescence intensity (normalized) in the channels during a period of 2 hours after the biofilms had reached the desired time point. Images are as follows: (A) 24 hours with DispersinB (B) 24 hours with water, (C) 12 hours with DispersinB, and (D) 12 hours with water.

reduced to $40.8 \%$ of its control, while A92 was reduced to $70.1 \%$, and SP102 was only slightly reduced to $96.1 \%$ using a 1:100 dilution of DispersinB-Gentamycin. This decline was more prominent using1:1 dilutions of DispersinBGentamycin; A12 was reduced to $11.9 \%$ of its control value, A92 was reduced to $35.5 \%$, and SP102 was reduced

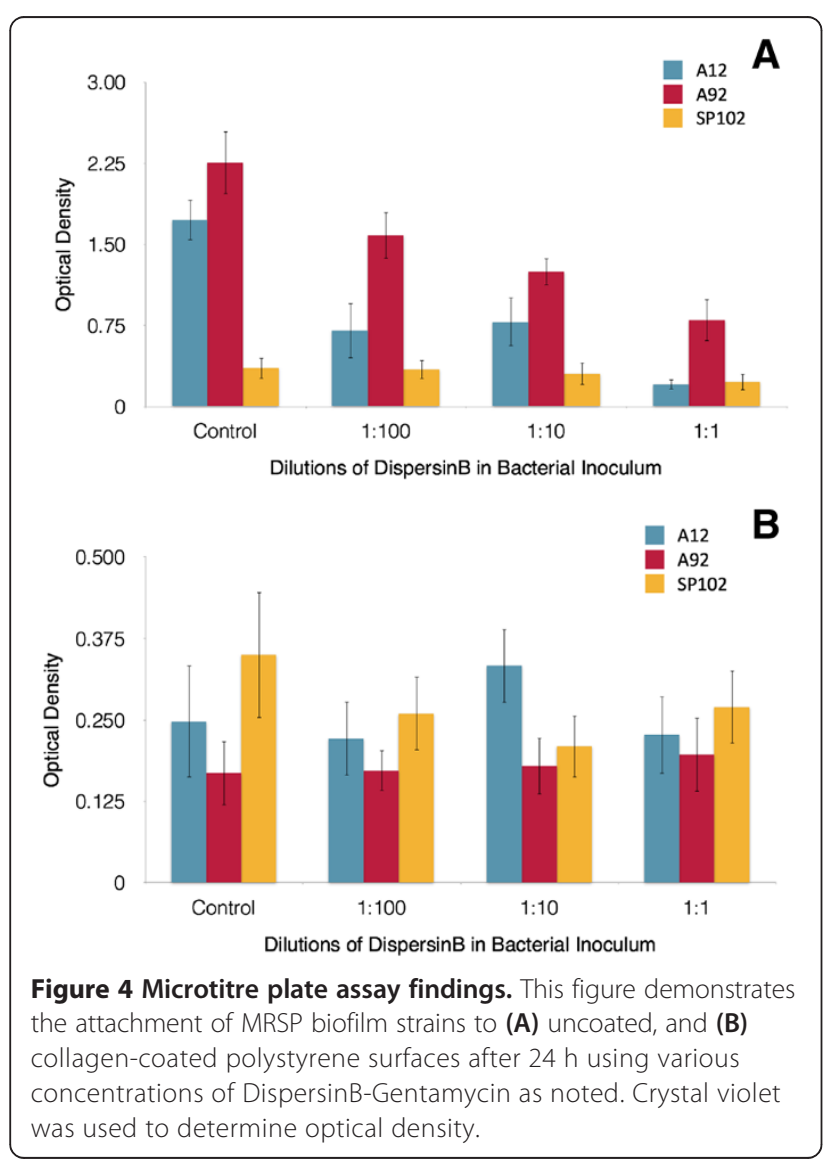

to $63.6 \%$. For collagen-coated plates (Figure 4B), the results showed a weaker correlation. Taking any error into account, each isolate showed a relatively stable level of biofilm density when comparing control values to all the concentrations of DispersinB-Gentamycin tested. The optical density values were much lower in collagen-coated tests (Figure $4 \mathrm{~B}$ ), with values routinely less than $0.5 \mathrm{com}$ pared to values that ranged up to an optical density of 2.258 in uncoated tests. Rat tail collagen was used for these studies, which is economical and readily available, but we acknowledge that this may not be the ideal substrate for MRSP attachment. In the future, we could test more relevant sources of collagen to determine if this improves overall biofilm formation.

\section{Conclusions}

Microfluidic tests performed as described in this study showed that there was minimal biofilm shearing from our microfluidic device, even following long periods of idle incubation. Although, we determined that the ability of DispersinB-Gentamycin to remove biofilm growth was not dramatically different than treatment with water, we contend that we have developed a successful model to study chronic wound infections in animals. Uncoated microtitre plate assays did confirm that DispersinBGentamycin is capable of effectively removing biofilm growth, which appears to be particularly effective against strains that are strong biofilm formers. Additional studies with our microfluidic device may be necessary to determine the best conditions for establishing a biofilm that resembles those that form during wound infection. We would suggest focusing on a strong biofilm forming strain, such as A12, possibly incorporating smaller channel widths or other sources or preparations of collagen. Wound infections are typically polymicrobial [21]. Hence 
co-culture studies may more accurately reflect the conditions present in vivo. We also would consider incorporating host cells into our model, such as endothelial cells. Ultimately, these results are preliminary, but we have developed a base model that may be modified to better emulate the conditions experienced in vivo during a chronic wound infection. Our model is easy to use, space-saving, and economical. Such a model may be highly effective in mimicking a wound infection without resorting to invasive testing of antimicrobial therapies in animals with wound and surgical site infections.

\section{Competing interests}

The authors declare that they have no competing interests.

\section{Author's contributions}

JT and SN designed the project. JT performed the experiments and wrote the manuscript with SN. SN contributed to the conceptual design and manuscript preparation. Both authors read and approved the manuscript.

\section{Acknowledgements}

The authors thank Dr. Scott J. Weese of the Ontario Veterinary College for providing bacterial strains and for his insightful discussions during the course of this project. This work was supported by funding from the Natural Sciences and Engineering Research Council of Canada, the Canadian Institutes for Health Research, and the Canada Foundation for Innovation. The authors sincerely thank Dr. Srinivasa Madhyastha of Kane Biotech for providing the DispersinB-Gentamycin samples.

Received: 27 November 2013 Accepted: 7 January 2014

Published: 13 January 2014

\section{References}

1. Frank LA, Loeffler A: Meticillin-resistant Staphylococcus pseudintermedius: clinical challenge and treatment options. Vet Dermatol 2012, 23:283-291.

2. Bryan J, Frank LA, Rohrbach BW, Burgette L, Cain CL, Bemis DA: Treatment outcome of dogs with meticillin-resistant and meticillin-susceptible Staphylococcus pseudintermedius pyoderma. Vet Dermatol 2012, 23:361-368

3. van Duijkeren E, Catry B, Greko C, Moreno MA, Pomba MC, Pyorala S, Ruzauskas M, Sanders P, Threlfall EJ, Torren-Edo J, Torneke K, A. Scientific Advisory Group on Antimicrobials (SAGM): Review on methicillin-resistant Staphylococcus pseudintermedius. J Antimicrob Chemother 2011, 66:2705-2714.

4. Daeschlein G: Antimicrobial and antiseptic strategies in wound management. Int Wound J 2013, 10:9-14.

5. Zhang $\mathrm{M}, \mathrm{Li} \mathrm{H}, \mathrm{Ma} \mathrm{H}$, Qin J: A simple microfluidic strategy for cell migration assay in an in vitro wound-healing model. Wound Repair Regen 2013, 21:897-903.

6. Larko E: Development of an in vitro model for testing novel antimicrobial substances: effects of established wound bacterial biofilm on cultured human macrophages, In Chemical and Biological Engineering. Gothenburg, Sweden: Chalmers Unviersity of Technology; 2011.

7. Torkian BA, Yeh AT, Engel R, Sun CH, Tromberg BJ, Wong BJ: Modeling aberrant wound healing using tissue-engineered skin constructs and multiphoton microscopy. Arch Facial Plast Surg 2004, 6:180-187.

8. Werthen $M$, Henriksson L, Jensen PO, Sternberg C, Givskov M, Bjarnsholt T: An in vitro model of bacterial infections in wounds and other soft tissues. APMIS 2010, 118:156-164.

9. Neethirajan S, Karig D, Kumar A, Mukherjee P, Retterer ST, Doktycz MJ: Biofilms in microfluidic devices. In Encyclopedia of Nanotechnology. Edited by Bhushan B. New York: Springer; 2012:213-219.

10. Chen CH, Lu Y, Sin ML, Mach KE, Zhang DD, Gau V, Liao JC, Wong PK: Antimicrobial susceptibility testing using high surface-to-volume ratio microchannels. Anal Chem 2010, 82:1012-1019.

11. Sato K, Sasaki N, Svahn HA, Sato K: Microfluidics for nano-pathophysiology. Adv Drug Deliv Rev 2013. doi: 10.1016/j.addr.2013.08.009.
12. Halder P, Nasabi M, Lopez FJ, Jayasuriya N, Bhattacharya S, Deighton M, Mitchell A, Bhuiyan MA: A novel approach to determine the efficacy of patterned surfaces for biofouling control in relation to its microfluidic environment. Biofouling 2013, 29:697-713.

13. Kim J, Park HD, Chung S: Microfluidic approaches to bacterial biofilm formation. Molecules 2012, 17:9818-9834.

14. Brindle ER, Miller DA, Stewart PS: Hydrodynamic deformation and removal of Staphylococcus epidermidis biofilms treated with urea, chlorhexidine, iron chloride, or DispersinB. Biotechnol Bioeng 2011, 108:2968-2977.

15. Dicicco M, Neethirajan S, Singh A, Weese JS: Efficacy of clarithromycin on biofilm formation of methicillin-resistant Staphylococcus pseudintermedius. BMC Vet Res 2012, 8:225.

16. Lee JH, Kaplan JB, Lee WY: Microfluidic devices for studying growth and detachment of Staphylococcus epidermidis biofilms. Biomed Microdevices 2008, 10:489-498.

17. Stepanovic S, Vukovic D, Hola V, Di Bonaventura G, Djukic S, Cirkovic I, Ruzicka F: Quantification of biofilm in microtiter plates: overview of testing conditions and practical recommendations for assessment of biofilm production by staphylococci. APMIS 2007, 115:891-899.

18. Gawande PV, Yakandawala N, Vetri KL, Madhyashtha S: In vitro antimicrobial and antibiofilm activity of DispersinB-Tricolsan wound gel against chronic wound-associated bacteria. Open Antimicrob Agents J 2011, 3:12-16.

19. Osland AM, Vestby LK, Fanuelsen H, Slettemeas JS, Sunde M: Clonal diversity and biofilm-forming ability of methicillin-resistant Staphylococcus pseudintermedius. J Antimicrob Chemother 2012, 67:841-848.

20. Liu Y, Tay JH: Metabolic response of biofilm to shear stress in fixed-film culture. J App/ Microbiol 2001, 90:337-342

21. Rotstein OD, Pruett TL, Simmons RL: Mechanisms of microbial synergy in polymicrobial surgical infections. Rev Infect Dis 1985, 7:151-170.

doi:10.1186/1477-3155-12-1

Cite this article as: Terry and Neethirajan: A novel microfluidic wound model for testing antimicrobial agents against Staphylococcus pseudintermedius biofilms. Journal of Nanobiotechnology 2014 12:1.

\section{Submit your next manuscript to BioMed Central and take full advantage of:}

- Convenient online submission

- Thorough peer review

- No space constraints or color figure charges

- Immediate publication on acceptance

- Inclusion in PubMed, CAS, Scopus and Google Scholar

- Research which is freely available for redistribution 